\title{
Mid-Span Spectral Inversion for Coherent Optical OFDM Systems: Fundamental Limits to Performance
}

\author{
Monir Morshed, Liang Bangyuan Du, Member, IEEE, and Arthur James Lowery, Fellow, IEEE
}

\begin{abstract}
We develop a theoretical expression to predict the ultimate back-to-back performance of coherent optical orthogonal frequency-division multiplexing (CO-OFDM) systems that rely on four-wave mixing to achieve phase conjugation for mid-span spectral inversion. Our analysis shows that two different two-stage nonlinear processes produce strong noise-like products in the conjugated signal band. We verify our theoretical results with simulations and experiments; these both show excellent agreement with the analytical theory. We identify the optimal design parameters and predict that optical phase conjugation of $10 \mathrm{THz}$ wide orthogonal frequency-division multiplexing signals could be possible, given appropriate dispersion management of the nonlinear element. We also experimentally demonstrate the benefit of MSSI in an $800 \mathrm{~km}$ transmission of CO-OFDM.
\end{abstract}

Index Terms-Coherent optical orthogonal frequency-division multiplexing (CO-OFDM) systems, mid-span spectral inversion (MSSI), optical phase conjugation (OPC), Kerr nonlinearity.

\section{INTRODUCTION}

$\mathbf{O}$ PTICAL orthogonal frequency-division multiplexing (OFDM) systems are a possible solution to meet the demand of $100 \mathrm{~Gb} / \mathrm{s}$ Ethernet data traffic and beyond [1]-[4]. Recent results have demonstrated Tbit/s rates over $600 \mathrm{~km}$ [5]. However, long-haul optical systems are affected by fiber nonlinearity unless the optical powers are kept low [6]. This constrains the design of long-haul systems; for example, low launch powers limit the optical signal-to-noise ratio (OSNR) that can be achieved for a given link, thereby limiting the size of the constellation that can be used [7]. Therefore, it is very important to mitigate fiber nonlinearity to achieve higher spectral efficiencies in long-haul transmission [7], [8].

Theoretical studies have suggested that the transmission performance can be significantly improved if ideal fiber nonlinearity mitigation techniques are used [9], such as digital backpropagation (BP) [10]-[12]. However, the high computational complexity of BP means that achieving ideal fiber nonlinearity compensation is impractical in a link using wavelength division

Manuscript received July 04, 2012; revised October 29, 2012; accepted November 04, 2012. Date of publication November 15, 2012; date of current version December 17, 2012. This work was supported by the Australian Research Council Centre of Excellence for Ultrahigh Bandwidth Devices for Optical Systems under Project CE110001018.

The authors are with the Centre of Excellence for Ultrahigh Bandwidth Devices for Optical Systems, Department of Electrical and Computer Systems Engineering, Monash University, Melbourne, Vic. 3800, Australia (e-mail: mohammad.monir@monash.edu; liang.du@monash.edu; arthur. lowery@monash.edu).

Color versions of one or more of the figures in this paper are available online at http://ieeexplore.ieee.org.

Digital Object Identifier 10.1109/JLT.2012.2227942 multiplexing (WDM) [11], [13]. Less computationally intensive SPM [14], [15] and XPM [16]-[18] compensation methods using DSP have been proposed. However, these methods are less effective for links without dispersion compensation [14], [18], therefore are less desirable for greenfield deployments.

Mid-span spectral inversion (MSSI), which uses optical phase conjugation (OPC) near the middle of the link, has been shown to be effective for nonlinearity compensation for intensity modulated systems [19], [20]. An advantage of using MSSI over electronic nonlinearity compensation is that it can process many WDM channels simultaneously [19], although at the expense of an added module in the outside plant. Although MSSI fell from favor due to its complexity, because systems are now constrained by the nonlinear Shannon limit [7], [8] and require higher OSNRs to support higher order modulation formats, MSSI may again become a useful technology in optical systems.

Recently, simulation results using MSSI with coherent optical OFDM (CO-OFDM) have been reported [21]. We have reported an experimental demonstration of fiber nonlinearity compensation using MSSI for a CO-OFDM superchannel [22], [23]. Pechenkin and Fair [24] presented a theoretical analysis of CO-OFDM systems using MSSI. They assumed an ideal MSSI module that only conjugates the incoming light to its output, and did not consider any other conjugation mechanisms that could degrade the signal inside the module.

In this paper, we present a detailed theoretical analysis of the performance limit of CO-OFDM systems that use MSSI. In Section II, we identify two different two-stage mixing mechanisms that limit the signal quality at high powers. The dominant limiting factor is the conjugated replica of the cross phase modulation (XPM) products between the pump and signal. The other significant limiting factor is the conjugated replica of the four-wave mixing (FWM) products of subcarriers. In Section III, we verify our theoretical analysis with split-step Fourier method (SSFM) simulations, which show excellent agreement with the analysis. We then use an experiment to further confirm the theory. In Section IV, we experimentally demonstrate transmission of four quadrature-amplitude modulation (4-QAM) with a data rate of $14.7 \mathrm{~Gb} / \mathrm{s}$ over 800 $\mathrm{km}$ to show that OPC could be used to reduce the effects of fiber nonlinearity in CO-OFDM systems, to improve system performance and transmission distance.

\section{THEORY}

\section{A. System Description}

To identify the negative impact of an MSSI module on signal quality, we consider a back-to-back system, as shown in 
(a)

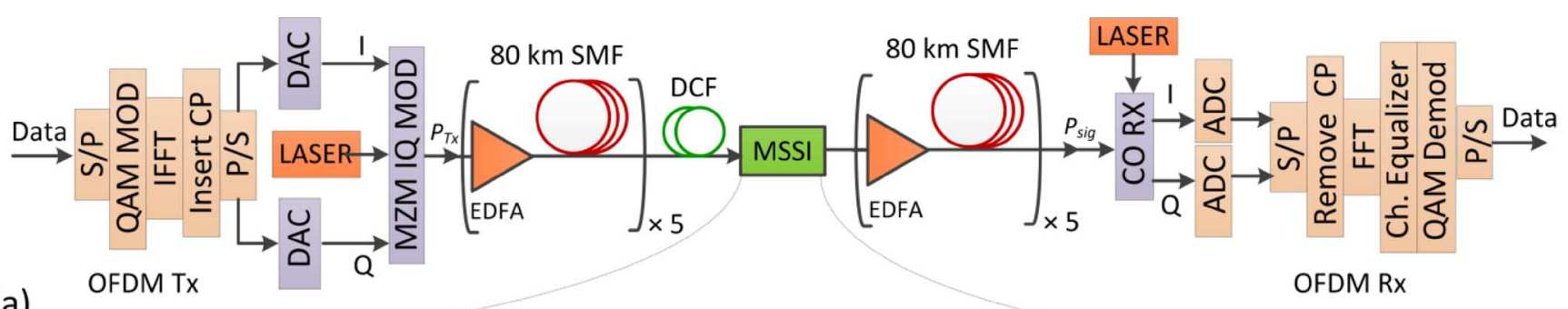

MSSI

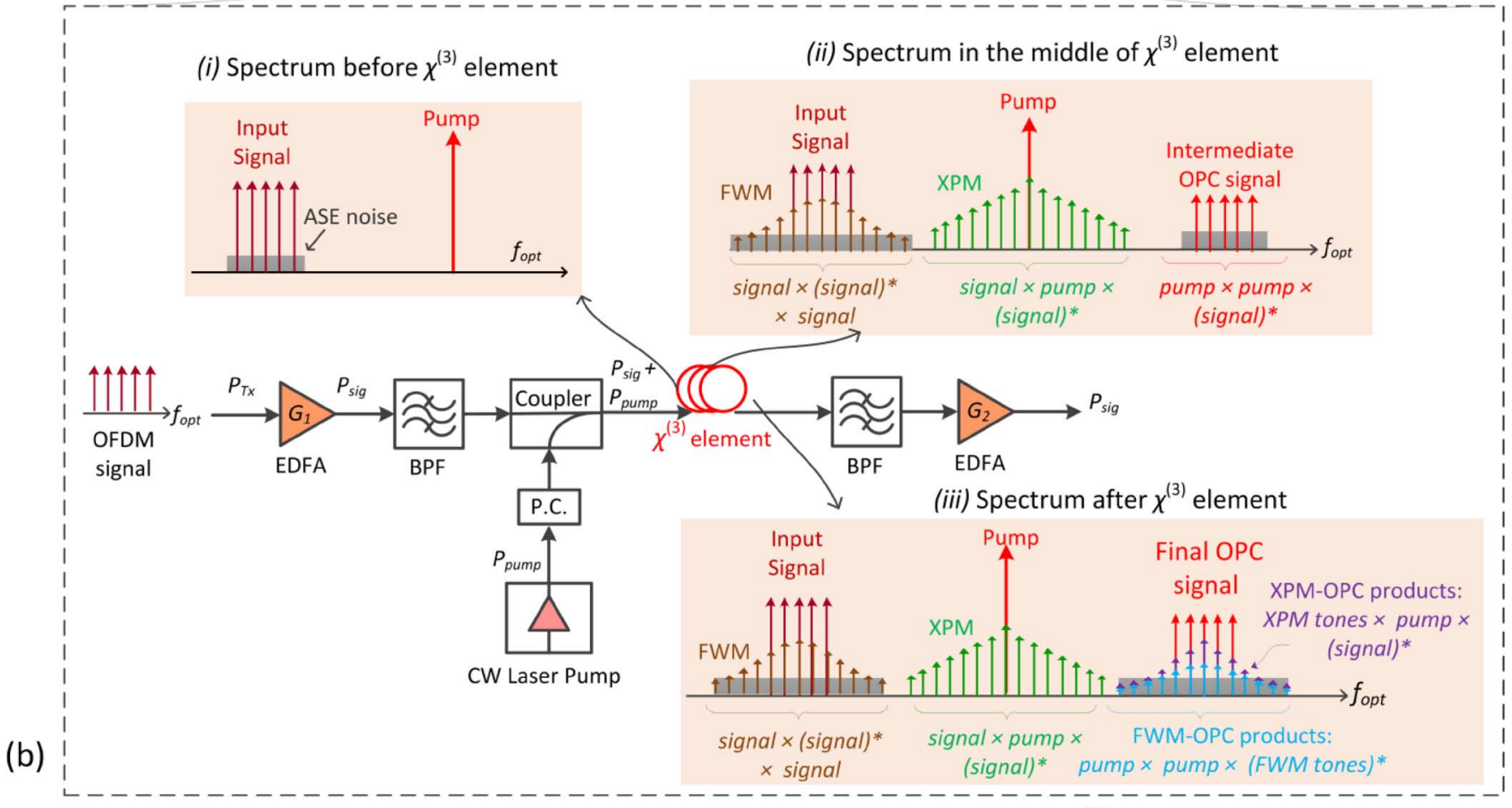

Fig. 1. System schematic and the spectrum at the input, middle, and output of the $\chi^{(3)}$ element.

Fig. 1(a) but without fiber spans and dispersion compensating fiber (DCF). The OFDM signal is generated by digital processing, a serial to parallel converter (S/P) distributes the input data stream to a bank of QAM modulators, which provides amplitude and phase coefficients for the inverse fast Fourier transform (IFFT). The IFFT produces a waveform that is a superposition of the QAM-modulated subcarriers. A cyclic prefix (CP) is inserted and a parallel to serial converter $(\mathrm{P} / \mathrm{S})$ outputs the waveform samples sequentially, to two digital-to-analog converters (DACs), which feed a complex optical modulator (MZM IQ MOD). The output of the optical modulator, at a power $P_{\mathrm{TX}}$, is fed into the MSSI module, bypassing the link.

The details of MSSI module are shown in Fig. 1(b). The input amplifier boosts the transmitter power, $P_{\mathrm{TX}}$. This input signal to the MSSI is then filtered with a bandpass filter (BPF) and is combined with the output of a continuous wave laser pump. In our simulation and theoretical analysis, the pump $\left(P_{\text {pump }}\right)$ and signal $\left(P_{\text {sig }}\right)$ powers are defined at the input to $\chi^{(3)}$ nonlinear element. The output of the $\chi^{(3)}$ nonlinear element is passed through a filter to remove the pump and the original signal, but leave the optical phase conjugated (OPC) signal. The OPC signal is then amplified before passing to a CO-OFDM coherent receiver. The coherent receiver feeds a digital processor that re- moves the CP, performs a Fourier transform to separate the subcarriers, equalizes the phases of the channels, and them demodulates the subcarriers to recover the data in each subcarrier.

The insets in Fig. 1(b) show the spectra at three different points along the $\chi^{(3)}$ nonlinear element, which is a highly nonlinear fiber (HNLF) in this paper. The first inset $(i)$ shows the spectrum at the input. Only amplified spontaneous emission (ASE) (gray color) generated by the input amplifier is present at this point. The second inset (ii) shows the spectrum at the middle of the HNLF. It shows the subcarrier intermodulation products (brown) that are generated by FWM between the OFDM subcarriers. The input signal $P_{\text {sig }}$ has been phase conjugated the opposite side of the pump signal, which we call the OPC signal (red). XPM products (green), due to signal $\times$ pump $\times(\text { signal })^{*}$ mixing, fall around the pump, where * denotes conjugation. As the two signals in the XPM mixing come from the same light source, these tones (green) are nondegenerate FWM products. However, to distinguish these tones from the FWM products that fall upon the signal's bandwidth (brown), we prefer to call these tones XPM. The third inset (iii) shows the spectrum at the output. It shows two additional features other than the ASE, which we call XPM-OPC products (purple) and FWM-OPC products (blue) falling over the conjugated 
signal. The XPM-OPC products are generated by nondegenerate FWM between XPM tones $\times$ pump $\times(\text { signal })^{*}$. The FWM-OPC products are generated due to degenerate FWM between pump $\times$ pump $\times(\text { FWM tones })^{*}$. The XPM-OPC and FWM-OPC products fall partially on the conjugated signal's band (red), so cannot be filtered out by the second BPF. Together we call these two new features $O P C$ distortions. Strictly, these are deterministic distortions, though, due to the large numbers of modulated subcarriers, these will have noise-like properties. In the following sections, we calculate the power of the OPC signal and all the aforementioned relevant distortions separately to determine the system's $Q$. The analysis has been done for a single-polarization case, where all interacting wavelengths (signal and pump) are copolarized. The effect of cross-polarization modulation is not considered.

\section{B. Calculation of OPC Distortion Power}

The derivation of OPC distortion power follows that of [25], which gives a simple method of counting the numbers of distortion products and calculating their powers. It relies on each product growing coherently along the fiber; if their fields increase linearly, their powers will grow quadratically. At the receiver, the products falling on a particular subcarrier signal are mostly incoherent, so the error they cause is proportional to the sum of their powers. However, in the nonlinear element, the OPC products are generated by a two-stage process: 1) the FWM or XPM products are generated; 2) they are phase conjugated to fall on the conjugated signal band. The analysis of [25] only accounted for products formed by the first stage; therefore, it must be extended to calculate the power of the two-stage mixing products.

Lowery et al. [25] provide a simple equation describing the output power of a nonlinear product, $P_{i j k}$, due to three tones, powers $P_{i}, P_{j}, P_{k}$, propagating through a lossless and dispersionless nonlinear element of length $\Delta z$. This is

$$
P_{i j k}=\left(\frac{D_{a}}{3} \gamma \Delta z\right)^{2} P_{i} P_{j} P_{k}
$$

where $D_{a}$ is the degeneracy factor which equals 6 for nondegenerate (NDG) products and 3 for degenerate (DG) products, and $\gamma$ is the nonlinear coefficient.

1) Calculation for FWM-OPC Products: We shall first analyze the FWM-OPC products; the process by which they are generated is illustrated in Fig. 2. The calculations assume $P_{\text {pump }}$ is not depleted by the nonlinear process. The first stage is that one of many FWM products is generated by three input subcarriers (indices $i, j, k$ ), each of power $P_{\mathrm{SC}}$, undergoing FWM (two in the degenerate case). This process occurs from the input of the HNLF to its output. Using (1), in each incremental length, $\Delta z$, of the fiber, at position $z_{1}$ along the fiber, the magnitude of the FWM field generated (watts ${ }^{0.5}$ ) is

$$
\left|\Delta E_{\mathrm{FWM}}\left(z_{1}\right)\right|=\frac{D_{a}}{3} \gamma \Delta z \sqrt{P_{\mathrm{SC}}\left(z_{1}\right) P_{\mathrm{SC}}\left(z_{1}\right) P_{\mathrm{SC}}\left(z_{1}\right)} .
$$

If we assume that the nonlinear element is lossless, then $P_{\mathrm{SC}}\left(z_{1}\right)$ becomes the input power of each subcarrier, $P_{\mathrm{SC}}(0)$, which we

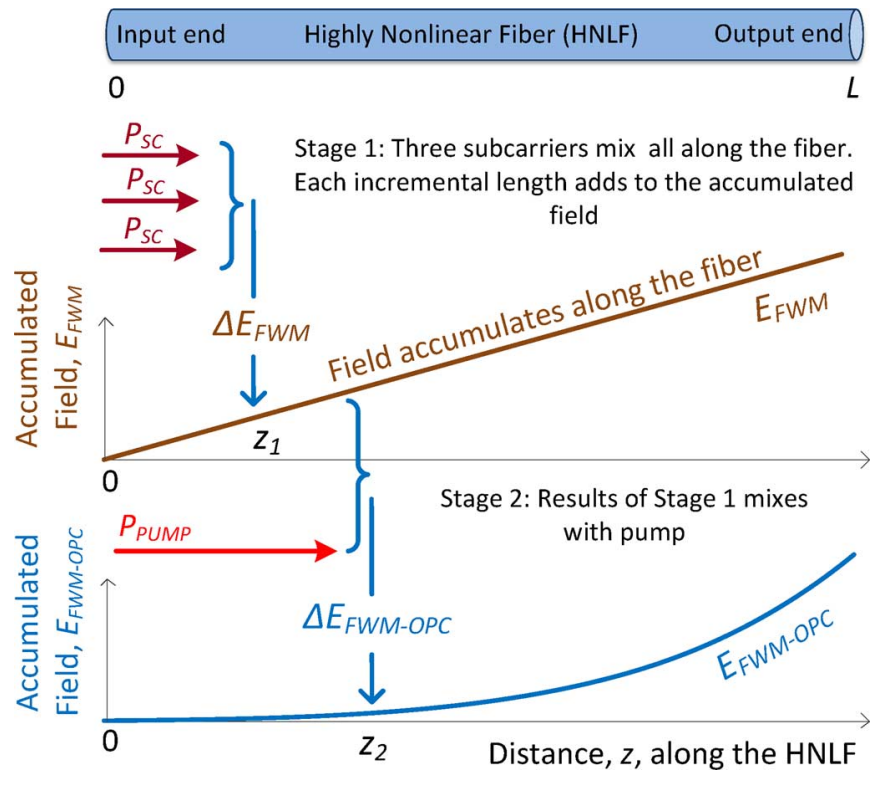

Fig. 2. Two-stage calculation process of a single FWM-OPC product's field: Stage 1-FWM between subcarriers; Stage 2-Conjugation due to mixing of the result of Stage 1 with the pump.

shorten to $P_{\mathrm{SC}}$. In the limit of $\Delta z \rightarrow 0$, the growth rate of the field is

$$
\frac{d\left|E_{\mathrm{FWM}}\left(z_{1}\right)\right|}{d z}=\frac{D_{a}}{3} \gamma \sqrt{P_{\mathrm{SC}}^{3}}
$$

The field at distance $z_{1}$ along the nonlinear element is found by integrating (3), which gives

$$
\left|E_{\mathrm{FWM}}\left(z_{1}\right)\right|=\frac{D_{a}}{3} \gamma z_{1} \sqrt{P_{\mathrm{SC}}^{3}} .
$$

Thus, the magnitude of the field associated with one FWM product (one combination of $i, j$ and $k$ ) grows linearly with distance along the fiber, as shown in the brown trace in Fig. 2.

Stage 2 of the process for FWM-OPC mixes the accumulated product $E_{\mathrm{FWM}}\left(z_{1}\right)$ with the pump $\times$ pump, at every point along the HNLF. We again assume the HNLF is lossless, so the pump power is constant along its length. At $z_{2}$, a new field is created, and is given by

$$
\left|\Delta E_{\mathrm{FWM}-\mathrm{OPC}}\left(z_{2}\right)\right|=\frac{D_{b}}{3} \gamma \Delta z\left|E_{\mathrm{FWM}}\left(z_{2}\right)\right| P_{\mathrm{pump}} .
$$

$D_{b}$ is the degenerate factor in this case. If we substitute in from (4), we find that the incremental contributions to the product's field are dependent upon the distance along the fiber, as

$$
\left|\Delta E_{\mathrm{FWM}-\mathrm{OPC}}\left(z_{2}\right)\right|=\frac{D_{a} D_{b}}{9}\left(\gamma z_{2}\right)(\gamma \Delta z) \sqrt{P_{\mathrm{SC}}^{3}} P_{\mathrm{pump}} .
$$

We can find the growth rate of this field at any point $z$ using the limit $\Delta z \rightarrow 0$

$$
\frac{d\left|E_{\mathrm{FWM}-\mathrm{OPC}}(z)\right|}{d z}=\frac{D_{a} D_{b}}{9}(\gamma z) \gamma \sqrt{P_{\mathrm{SC}}^{3}} P_{\mathrm{pump}} .
$$


By integration over the length of the HNLF, we find that the field at the output of the HNLF is

$$
\left|E_{\mathrm{FWM}-\mathrm{OPC}}(L)\right|=\frac{D_{a} D_{b}}{9} \frac{(\gamma L)^{2}}{2} \sqrt{P_{\mathrm{SC}}^{3}} P_{\mathrm{pump}} .
$$

The factor of $1 / 2$ that comes from the integration is physically due to the second process being vanishingly small at the start of the fiber, because the first process has yet to begin. We call this factor the two-stage effective length factor.

This calculation has provided the field strength of a single FWM-OPC product. There will be in the order of $N_{\mathrm{SC}}$-cubed of these products, due to the various combinations of subcarriers $(i, j, k)$ contributing to the mixing process. Many of these products will fall on the same conjugated subcarrier, so we will define that $N_{\mathrm{FWM}-\mathrm{OPC}}\left(k^{\prime}\right)$ fall on conjugated subcarrier $k^{\prime}$. Because the original subcarriers are phase modulated, the products falling on a particular subcarrier will be incoherent, so their powers will add. Also, in the majority of cases, $D_{a}=6$. Thus, the total power of the FWM-OPC products falling on a single conjugated subcarrier $k^{\prime}$ will be

$$
P_{\mathrm{FWM}-\mathrm{OPC}}\left(k^{\prime}\right)=N_{\mathrm{FWM}-\mathrm{OPC}}\left(k^{\prime}\right)(\gamma L)^{4} P_{\mathrm{SC}}^{3} P_{\text {pump }}^{2} .
$$

2) Calculation of XPM-OPC Products: A similar calculation can be performed for the XPM-OPC products, as shown in Fig. 3. At Stage 1, the incremental field is

$$
\left|\Delta E_{\mathrm{XPM}}(z)\right|=\frac{D_{c}}{3} \gamma \Delta z \sqrt{P_{\mathrm{SC}} P_{\mathrm{SC}} P_{\mathrm{pump}}} .
$$

$D_{c}$ is the nondegenerate factor in this case. The field of a single XPM product grows linearly along the fiber and at distance $z_{1}$ is

$$
\left|E_{\mathrm{XPM}}\left(z_{1}\right)\right|=\frac{D_{c}}{3} \gamma z_{1} \sqrt{P_{\mathrm{SC}} P_{\mathrm{SC}} P_{\mathrm{pump}}} .
$$

Stage 2 mixes the above field with an original subcarrier and the pump at every point along the fiber. The rate of growth of the resultant XPM-OPC product is

$$
\frac{d\left|E_{\mathrm{XPM}-\mathrm{OPC}}(z)\right|}{d z}=\frac{D_{c} D_{d}}{9}(\gamma z) \gamma \sqrt{P_{\mathrm{SC}}^{3}} P_{\mathrm{pump}} .
$$

Both $D_{c}$ and $D_{d}$ are nondegenerate factors. Integrating along the whole length $L$ of the HNLF gives

$$
\left|E_{\mathrm{XPM-OPC}}(L)\right|=\frac{D_{c} D_{d}}{9} \frac{(\gamma L)^{2}}{2} \sqrt{P_{\mathrm{SC}}^{3}} P_{\text {pump }} .
$$

Again, because the products from many combinations of input subcarrier are incoherent, the power of the tones that all fall on the same conjugated subcarrier index $k^{\prime}$ is

$$
P_{\mathrm{XPM}-\mathrm{OPC}}\left(k^{\prime}\right)=4 N_{\mathrm{XPM}-\mathrm{OPC}}\left(k^{\prime}\right)(\gamma L)^{4} P_{\mathrm{SC}}^{3} P_{\mathrm{pump}}^{2}
$$

where $N_{\mathrm{XPM} \text {-OPC }}\left(k^{\prime}\right)$ is the number of the XPM-OPC products falling on conjugated subcarrier $k^{\prime}$. Note how $P_{\mathrm{XPM}-\mathrm{OPC}}$ is four times stronger than $P_{\mathrm{FWM}-\mathrm{OPC}}$ because the pump was degenerate in Stage 2 of the calculation of $P_{\mathrm{FWM}-\mathrm{OPC}}$.

3) Calculation of the Numbers of Products Falling on a Conjugated Subcarrier k': We shall now consider the exact number

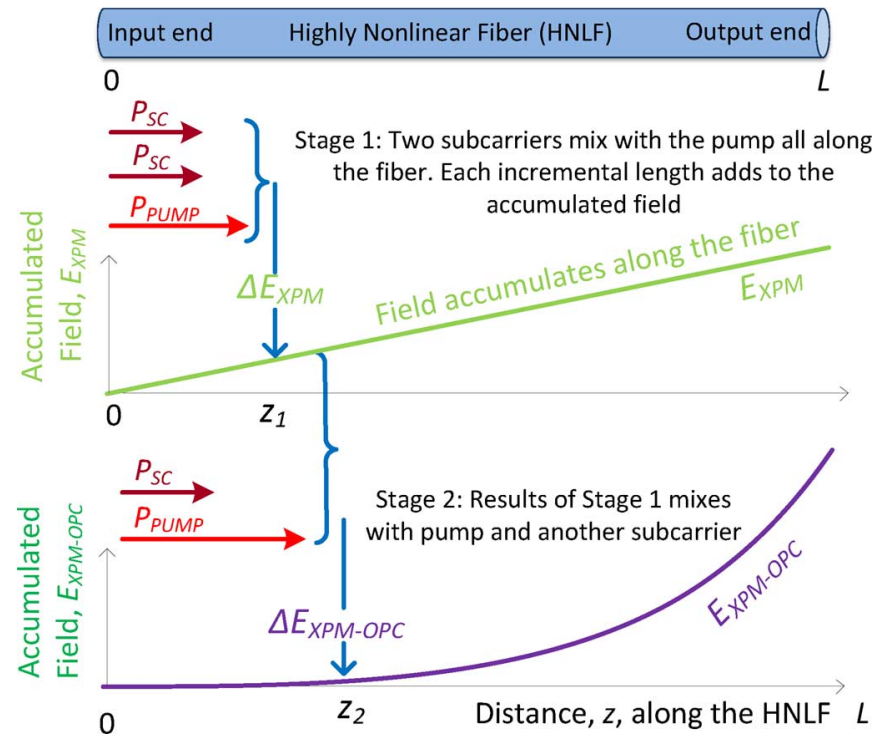

Fig. 3. Two-stage calculation process of a single XPM-OPC product's field: Stage 1 -XPM between subcarriers and pump; Stage 2-Conjugation due to mixing of the result of Stage 1 with the pump and another subcarrier.

of FWM-OPC products falling on a subcarrier. Lowery et al. [25] provided equations for the numbers of nondegenerate and degenerate products due to FWM of three (or two) subcarriers. As illustrated in Fig. 1 (ii) (brown), a proportion of these fall outside the bandwidth of the OFDM signal spectrum (dark red). These equations can be used for Stage 1 of the FWM-OPC process. The number of nondegenerate products is [25]

$$
N_{\mathrm{FWM}-\mathrm{NDG}}(k)=\frac{\left(3 N_{\mathrm{SC}}^{2}-10 N_{\mathrm{SC}}-4 k(k-1)\right)}{8}+1
$$

where $k$ is the index of the input subcarrier, over a range $-N_{\mathrm{SC}} / 2+1$ to $N_{\mathrm{SC}} / 2$. The number of degenerate products falling on a single subcarrier is

$$
N_{\mathrm{FWM}-\mathrm{DG}}(k)=\left(N_{\mathrm{SC}}-2\right) / 2 \text {. }
$$

$N_{\text {FWM-DG }}$ is a small number compared with the number of nondegenerate products subcarriers. Stage 2 simply mirrors these products around the pump, so there are the same numbers of products falling on the conjugated signals. In the simulations, we will calculate an average signal quality across the conjugated signals' band, so we can use an averaged number of products. Ignoring the degenerate products, this gives the average number of products per subcarrier as approximately [25]

$$
\overline{N_{\mathrm{FWM}-\mathrm{OPC}}} \approx 0.335 N_{\mathrm{SC}}^{2} \text {. }
$$

This result can be used with (9) to find the average power of the FWM-OPC products falling on a conjugated subcarrier, which is approximately

$$
\overline{P_{\mathrm{FWM}-\mathrm{OPC}}} \approx 0.335 N_{\mathrm{SC}}^{2}(\gamma L)^{4} P_{\mathrm{pump}}^{2} P_{\mathrm{SC}}^{3} .
$$

A similar calculation can be performed for the XPM-OPC power falling on a subcarrier; however, in Stage 1, there are 


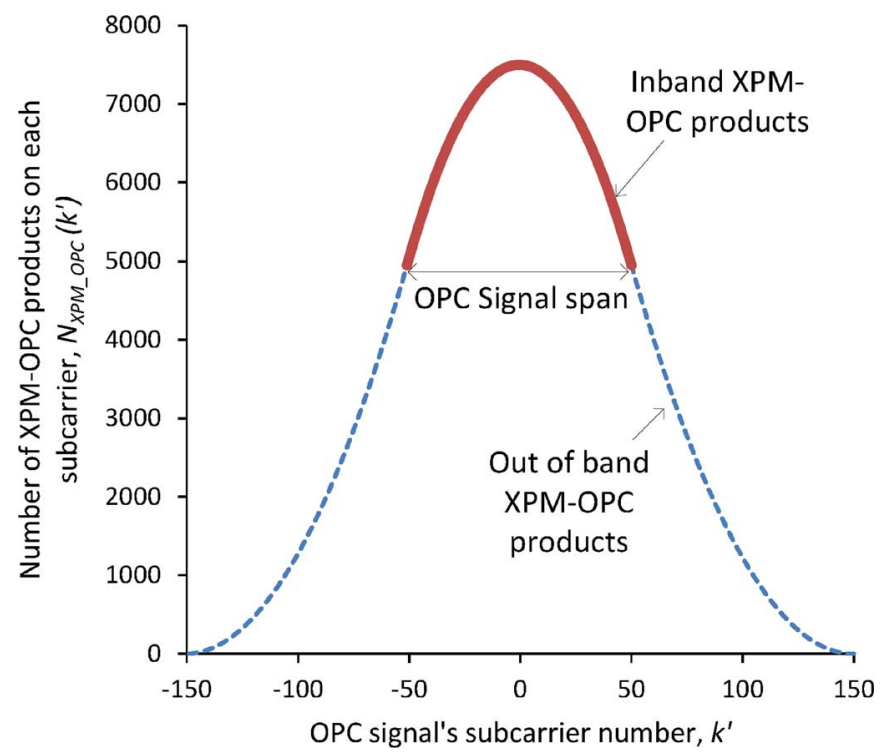

Fig. 4. Number of XPM-OPC products falling on and around the conjugated (OPC) signal bandwidth.

$N_{\mathrm{SC}}^{2}$ XPM products, as the pump frequency is fixed. Stage 2 mixes each of these products with the pump and $N_{\mathrm{SC}}$ of input subcarriers, so the number of possible combinations will be multiplied by $N_{\mathrm{SC}}$, giving a total of $N_{\mathrm{SC}}^{3}$ XPM-OPC products. Again, many of these products fall outside the band of the OPC signal. We used MATLAB to calculate the frequencies of all possible XPM-OPC products. Fig. 4 plots the number of XPM-OPC products falling on a particular subcarrier, $N_{\mathrm{XPM}-\mathrm{OPC}}\left(k^{\prime}\right)$. The number of subcarriers is 100 , which we used in both simulation and experiments described in Section III.

The ratio of the number of in-band XPM-OPC products to the total number of products was found to be $2 / 3$, so the average power of an in-band XPM-OPC product is found by multiplying (14) with factor of $2 / 3$ :

$$
\overline{P_{\mathrm{XPM}-\mathrm{OPC}}} \approx 2.667 N_{\mathrm{SC}}^{2}(\gamma L)^{4} P_{\mathrm{pump}}^{2} P_{\mathrm{SC}}^{3} .
$$

The average power of both types of in-band OPC products that fall on a conjugated subcarrier is the sum of (18) and (19), which gives

$$
\begin{aligned}
P_{\mathrm{OPC}} & =P_{\mathrm{XPM}-\mathrm{OPC}}+P_{\mathrm{FWM}-\mathrm{OPC}} \\
& =3 N_{\mathrm{SC}}^{2}(\gamma L)^{4} P_{\mathrm{pump}}^{2} P_{\mathrm{SC}}^{3} .
\end{aligned}
$$

\section{Power of a Conjugated Subcarrier}

Using (1) [25], the power of a single OPC subcarrier, $P_{\mathrm{SC}-\mathrm{OPC}}$, is

$$
P_{\mathrm{SC}-\mathrm{OPC}}=\frac{D_{\mathrm{DG}}^{2}}{9}(\gamma L)^{2} P_{\mathrm{pump}}^{2} P_{\mathrm{SC}}=(\gamma L)^{2} P_{\mathrm{pump}}^{2} P_{\mathrm{SC}}
$$

\section{Calculation of FWM Distortion Power}

Another type of distortion is caused by intermodulation between the subcarriers after they have been phase conjugated, which is caused by nondegenerate FWM. Again, this process occurs once a subcarrier has been phase-conjugated. Using our previously derived two-stage effective length factor of 0.5 to calculate the power of these distortions and a factor to account for some of these products falling out of band [25], we estimate that

$$
P_{\mathrm{FWM}}=0.335 N_{\mathrm{SC}}^{2}(\gamma L)^{2} P_{\mathrm{SC}-\mathrm{OPC}}^{3}
$$

\section{E. Calculation of ASE}

The ASE noise generated in the input amplifier is conjugated to fall upon the conjugate signal bandwidth. Added to this is the ASE noise from the second amplifier. If the gain factors of the amplifiers are $G_{1}$ and $G_{2}$, the ASE at the output of the second amplifier in a single polarization, within the bandwidth of a subcarrier $B_{\mathrm{SC}}$ is

$$
\begin{aligned}
P_{\mathrm{ASE}}= & 0.5 F \times B_{\mathrm{SC}} \times h f \\
& \times\left(\left(G_{1}-1\right)\left(\gamma L P_{\mathrm{pump}}\right)^{2} G_{2} L_{\mathrm{HNLF}}+\left(G_{2}-1\right)\right)
\end{aligned}
$$

where $F$ is the noise figure of the amplifiers, $h f$ is the energy of a photon, and $G_{1}$ and $G_{2}$ are the gain factors of the first and second amplifiers, respectively. In the simulations, $G_{1}$ is set to give the required $P_{\text {sig }}$ assuming a modulator output, $P_{\mathrm{TX}}$ of $-20 \mathrm{dBm}$, and $G_{2}$ is set to compensate for the loss in signal power during conjugation and the intrinsic loss factor of the HNLF $L_{\mathrm{HNLF}}$ found from (24), so

$$
G_{2}=\left(\left(\gamma L P_{\mathrm{pump}}\right)^{2} L_{\mathrm{HNLF}}\right)^{-1}
$$

where $L_{\mathrm{HNLF}}=10^{-\left(\alpha_{\mathrm{HNLF}} L\right) / 10}$.

\section{F. Calculation of the System $Q$}

For the sake of comparison, we calculate the average $Q$-value over all of the received subcarriers in both the simulations and theory. The average signal quality $q$ over all subcarriers, where $Q(\mathrm{~dB})=20 \cdot \log _{10}(q)$, the ratio of subcarrier power to all of the noise and distortions in the bandwidth of a subcarrier, given by

$$
q^{2}=\frac{G_{2} P_{\mathrm{SC}-\mathrm{OPC}}}{G_{2} P_{\mathrm{OPC}}+G_{2} P_{\mathrm{FWM}}+P_{\mathrm{ASE}}} .
$$

In the aforementioned equation, the OPC signal, FWM-OPC, and FWM-OPC terms have been multiplied by $G_{2}$ to make the output of the second erbium-doped fiber amplifier (EDFA) as the reference point for $Q$-value calculation.

The effect of attenuation in the nonlinear process can be approximately accounted for by using $L_{\text {eff }}$ instead of $L$ [25] in (20)-(22). However, there will be a small error because the two-stage effective length factor is $1 / 2$ only for the lossless case; loss slightly increases this as shown later in Fig. 9. A perfect model for a fiber with loss can be achieved by integrating along $z$ for the true power map. 
TABLE I

PARAMETER VAlues

\begin{tabular}{l|l}
\hline \multicolumn{1}{c|}{ Quantity } & \multicolumn{1}{c}{ Value } \\
\hline Noise Figure, $N F$ & $6 \mathrm{~dB}$ \\
Optical Frequency, $f$ & $193.1 \mathrm{THz}$ \\
Optical Bandwidth, $B_{o}$ & $7.8125 \mathrm{GHz}$ \\
Transmitter Output, $P_{T X}$ & $-20 \mathrm{dBm}$ \\
HNLF loss, $\alpha_{H N L F}$ & $0.97 \mathrm{~dB} / \mathrm{km}$ \\
HNLF Dispersion Coeff., CD & $0 \mathrm{ps} / \mathrm{nm} / \mathrm{km}$ \\
Length of the HNLF, $L$ & $1000 \mathrm{~m}$ \\
Nonlinear Coefficient, $\gamma$ & $11.5 \mathrm{~W}^{-1} \cdot \mathrm{km}^{-1}$ \\
Pump Power, $P_{\text {pump }}$ & $7.8 \mathrm{dBm}$ \\
& \\
\hline
\end{tabular}

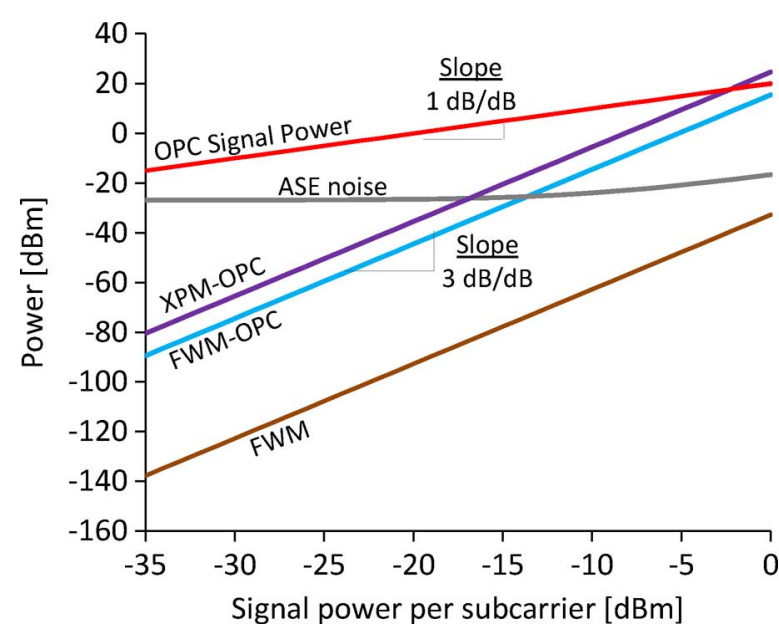

Fig. 5. OPC signal power and noise powers versus input signal subcarrier power (at the output of the output EDFA). The powers are summed over the bandwidth of the conjugated OFDM spectrum.

\section{G. System Performance}

We consider a hypothetical case with system parameters as given in Table I.

Fig. 5 shows the average powers of the unwanted products falling within the signal band and noise versus the input signal subcarrier power. The results show that ASE is the limiting factor below a subcarrier power of $-17 \mathrm{dBm}$ : XPM-OPC is the most severe limitation at powers above $-17 \mathrm{dBm}$. The power of FWM-OPC, which is $9 \mathrm{~dB}$ lower than the XPM-OPC, is also significant. The FWM products due to the OPC signal subcarriers are insignificant, being $60 \mathrm{~dB}$ below the $\mathrm{OPC}$ distortions. This is because the conjugated signals are about $20 \mathrm{~dB}$ below the input subcarriers.

Fig. 5 also shows that at an input subcarrier power of -3.0 $\mathrm{dBm}, \mathrm{XPM}-\mathrm{OPC}$ added to FWM-OPC becomes stronger than the OPC signal. This is because these terms increase with the cube of the signal power, whereas the OPC signal power only increases linearly with the signal power. Therefore, the usable signal power into the HNLF is limited by this effect. On the other hand, an EDFA is required to amplify the OPC signal after the nonlinear element, which produces ASE. These counteracting features imply that there is an optimal input signal power that maximizes back-to-back OPC performance.

Fig. 6 plots the calculated OPC signal power (-), total unwanted products $\left(P_{\mathrm{OPC}}\right.$ and $\left.\mathrm{ASE}\right)(-)$, and the $Q$-value (॰)

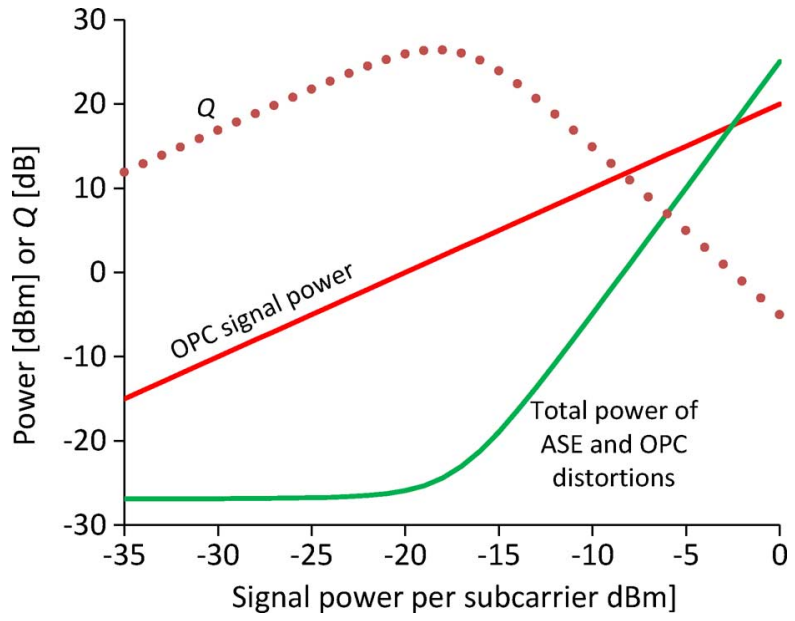

Fig. 6. Theoretical back-to-back performance of MSSI module versus input signal power per subcarrier.

against input signal subcarrier power. It shows that the optimum signal subcarrier power is $-18 \mathrm{dBm}$. An important conclusion from Fig. 6 is that an MSSI module can limit the maximum achievable performance of the system, even if the transmission fiber's nonlinearity is completely canceled by the MSSI module. For higher order modulation schemes, where a high $Q$-value is crucial, care should be taken to raise this limit by optimizing the MSSI module, by using high $P_{\text {pump }}$ and $\gamma L$, and also by operating with optimum signal power into the $\chi^{(3)}$ nonlinear element for the specific pump power.

\section{BACK-TO-BACK SimUlATION AND EXPERIMENTS}

In this section, the analytical results in Section 2 are compared with simulation and experimental results. The numerical simulations were conducted using VPItransmissionMaker v8.7. The OFDM signal was generated using MATLAB for the simulations and experiments, using a 128-point IFFT. 100 subcarriers were modulated with 4-QAM and eight-point $\mathrm{CP}$ was inserted.

The schematic of the experimental setup is shown in Fig. 1(a). However, in the back-to-back configuration, the fiber spans and the DCF have not been used. For the experiment, we used a Tektronix AWG 7102 10-GSamples/s two-port arbitrary waveform generator (AWG) in the transmitter. The inphase (I) and quadrature $(\mathrm{Q})$ components of the AWG drove a Sumitomo complex optical Mach-Zehnder modulator, modulating an Agilent external cavity laser (ECL), to create a $14.7 \mathrm{~Gb} / \mathrm{s}$ OFDM signal. A single-polarization coherent receiver, consisting of a Kylia optical hybrid and $\mathrm{U}^{2} \mathrm{~T}$ balanced photodiodes, was used to down convert the optical signal. A polarization controller was used to maximize the detected electrical power. A Photonetics Tunics ECL was used as the local oscillator. An Agilent Infiniium DSO-X 92804A 80-GSample/s 28-GHz real-time sampling oscilloscope was used as the analog to digital converter (ADC). The digital signal was then equalized using a typical single-tap OFDM equalizer [26], [27].

Fig. 7 shows the theoretical, simulated, and experimental $Q$-values when the input signal subcarrier power is varied. The simulation results match very well with the results obtained from the analytical calculation. The experimental results show 


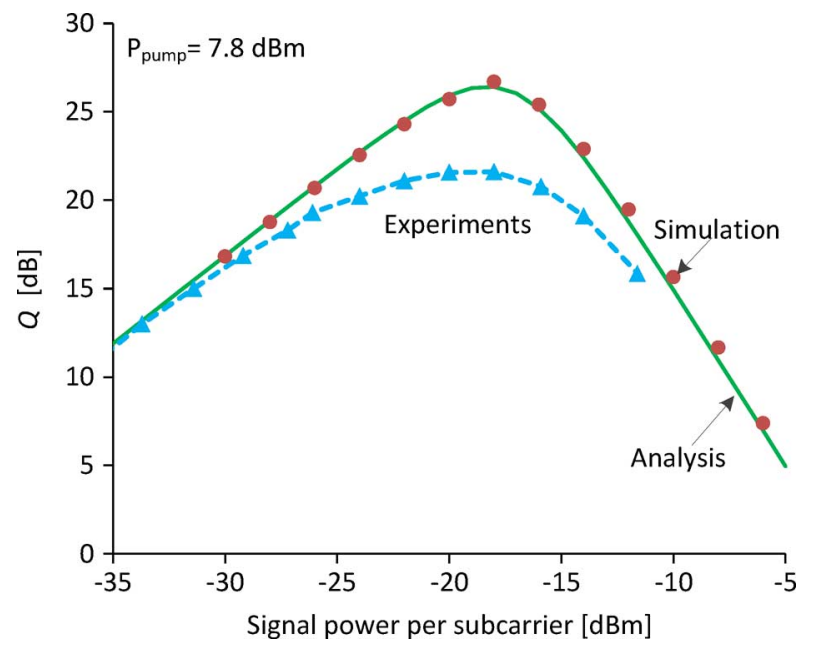

Fig. 7. Analytical (green solid line - ), simulated (red circles $\bullet$ ), and experimental (blue dashed lines with triangle $\Delta$ ) $Q$-values versus input signal subcarrier power for a back-to-back system.

a ceiling in the peak performance. This could be because of system imperfections such as the quantization noise of the DAC and ADC, imbalances in the I and Q signal paths, and laser phase noise.

We will now consider the effect of three design parameters: pump power, the length of the nonlinear element, and the total bandwidth of the optical signal.

\section{A. Influence of Pump Power}

Fig. 8 shows theoretical and simulated $Q$-value for three different pump powers $P_{\text {pump. }}$. It shows that the $Q$-value in the ASE region increases by $2 \mathrm{~dB}$ for every $1 \mathrm{~dB}$ increase in the pump power. This agrees with (23), since the OPC signal is proportional to $P_{\text {pump }} \times P_{\text {pump }} \times P_{\mathrm{SC}}$. At nonlinearity-limited input subcarrier powers, the $Q$-value does not increase by using higher pump powers. This is because the main limiting factor, the OPC distortion power, given by (20), is also proportional to $P_{\text {pump }} \times P_{\text {pump }}$. This causes both the OPC signal and the OPC distortion to increase with the square of $P_{\text {pump }}$; thus, the performance in the nonlinear region does not improve when $P_{\text {pump }}$ is increased.

Importantly, the optimum input signal subcarrier power at which the $Q$ peaks shifts toward lower powers when $P_{\text {pump }}$ is increased. Additionally, a simple geometrical calculation predicts that the $Q_{\max }$ is improved by $2 \sqrt{3} /(1+\sqrt{3})=1.26 \mathrm{~dB}$ for every $1 \mathrm{~dB}$ increase of $P_{\text {pump }}$.

\section{$B$. Influence of the Length of the HNLF}

Fig. 9 shows the effect of the length of the HNLF on optimal signal quality, and the input power used to obtain each optimum. As the length is increased, the optimum signal quality increases; however, beyond $1000 \mathrm{~m}$, the quality begins to reduce in the simulation, but not in the theory. This shows that simply substituting $L_{\mathrm{eff}}$ for the total HNLF length, to account for attenuation, is inaccurate for long HNLF when two-stage processes are present, but is sufficient for most situations.

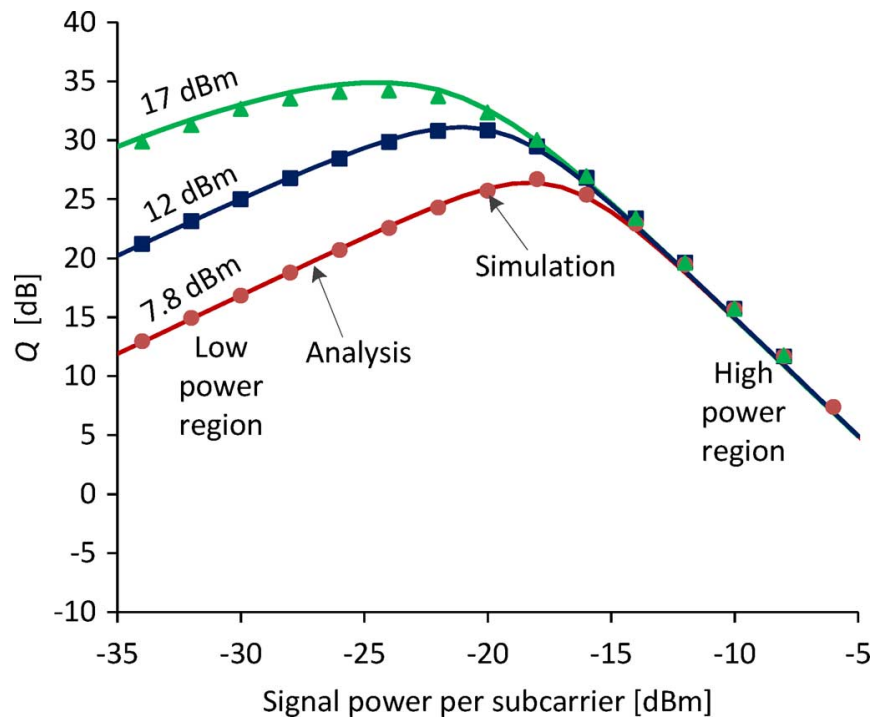

Fig. 8. Theoretical (solid lines) and simulated (symbols) $Q$-value versus signal subcarrier power for three pump powers.

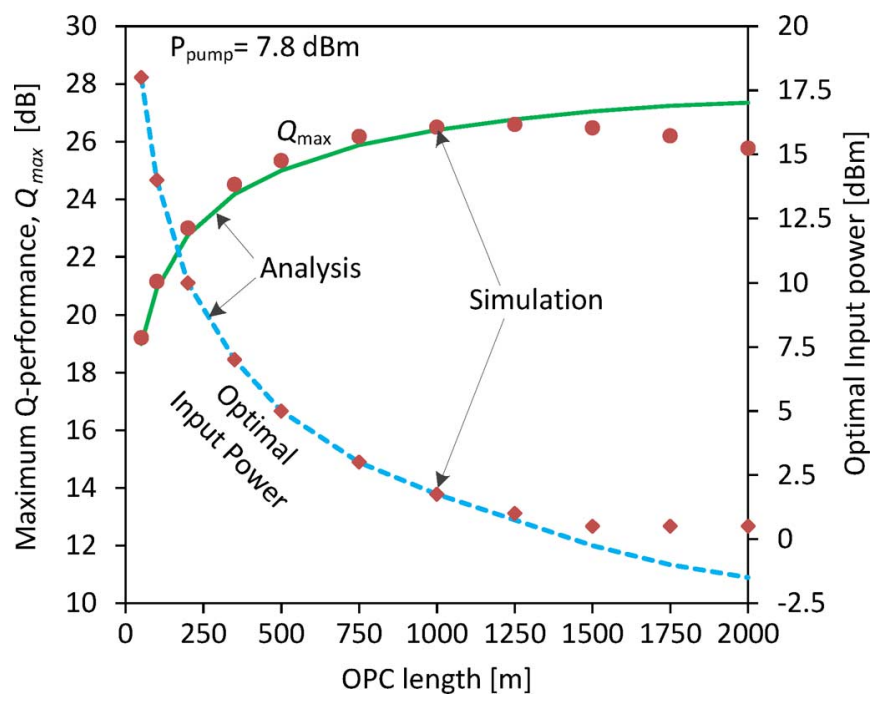

Fig. 9. Maximum $Q$ performance and optimal input power versus HNLF length.

\section{Influence of Signal Bandwidth}

Fig. 10 shows the simulation and analytical prediction of maximum $Q$-value for different optical OFDM signal bandwidths. The pump power was increased to $17 \mathrm{dBm}$ to accommodate the broadband OPC. The theoretical results agree very well with the simulation results irrespective of the OFDM signal bandwidth when $\mathrm{CD}$ is ignored; OPC could span the $S$, $C$, and $L$ bands $(\lambda=1460-1625 \mathrm{~nm}, \Delta f \approx 20 \mathrm{THz}$ ), provided the HNLF is dispersionless across the span or its dispersion is managed appropriately. However, simulation results using the parameters from our OFS HNLF $(\mathrm{CD}=0.01 \mathrm{ps} / \mathrm{nm} / \mathrm{km})$ show a penalty when the bandwidth is more than $1 \mathrm{THz}$.

\section{TRANSMISSION RESUlTS}

We built a CO-OFDM system with an MSSI module to identify any practical issues. Details of OFDM signal and data rate 


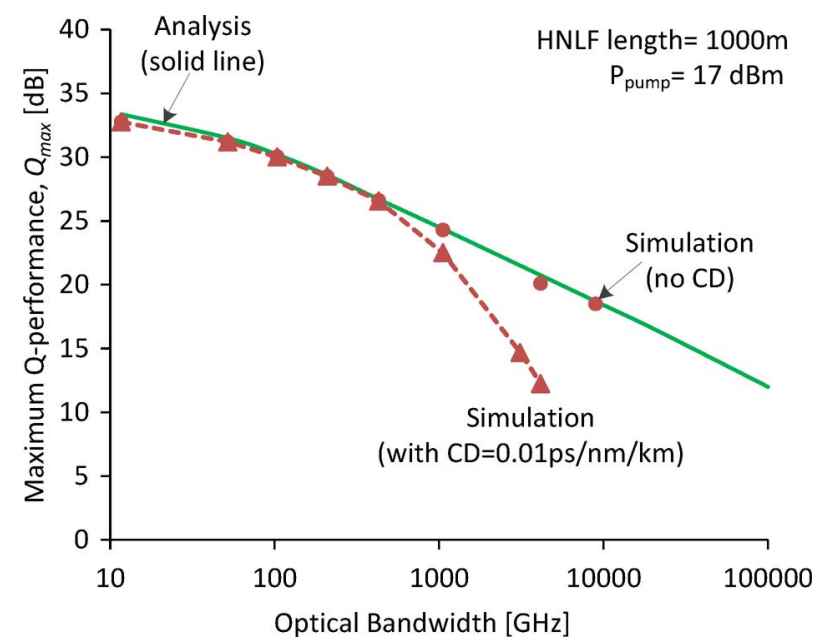

Fig. 10. Optimal signal quality and corresponding input power versus signal bandwidth.

are as described in Section III. The schematic of the transmission system is as in Fig. 1(a). The optical link comprised $10 \times 80$ $\mathrm{km}$ spans of S-SMF with EDFAs only to compensate the span loss. A DCF has been inserted just before the MSSI module to compensate $60 \mathrm{~km}$ of S-SMF's dispersion, which makes the system more symmetrical in terms of power against accumulated CD about the OPC [20]. However, it is clearly impossible to achieve perfect symmetry with only lumped amplifiers. The MSSI module is placed after the fifth span. Lightwaves 2020 forward-pumped EDFAs were used to set the launch power into the S-SMF spans. The launch powers into the S-SMF spans were swept to change the balance of ASE and fiber nonlinearity.

Fig. 1(b) shows the block diagram of the MSSI module used in the experiment. Here, the experimental setup will be explained in more detail. The signal was first amplified with an EDFA before being passed through a Siemens TransXpress Arrayed-Waveguide Router demultiplexer with a $200 \mathrm{GHz}$ passband centered on the signal, to remove the out-of-band ASE. The input signal was then combined with a pump from an Agilent Technologies N7714A Multiport Tunable Laser Source, tuned $1.8 \mathrm{~nm}$ lower than the signal's wavelength. The combined signal is coupled into $1000 \mathrm{~m}$ of OFS Inc. HNLF, which has properties, as described in Section III.

Fig. 11 shows the transmission results with and without the OPC. The experimental results show that the peak $Q$-value increases by $1 \mathrm{~dB}$ and the nonlinear threshold (NLT) power, which is the maximum launch power that supports a bit error rate $<10^{-3}(Q>9.7 \mathrm{~dB})$, also increases by $6 \mathrm{~dB}$ when the MSSI is used. The simulations show about $9 \mathrm{~dB}$ improvement in the NLT power. The simulations also show an increase in the maximum $Q$ by about $7 \mathrm{~dB}$.

For the case without the OPC, the experimental results show $2 \mathrm{~dB}$ better NLT power performance than the simulation results. The launch power for the experimental systems was set by operating the EDFAs in automatic power control mode. Since the measured power also contains ASE, the actual signal power will be lower than the recorded power. This makes the experimental

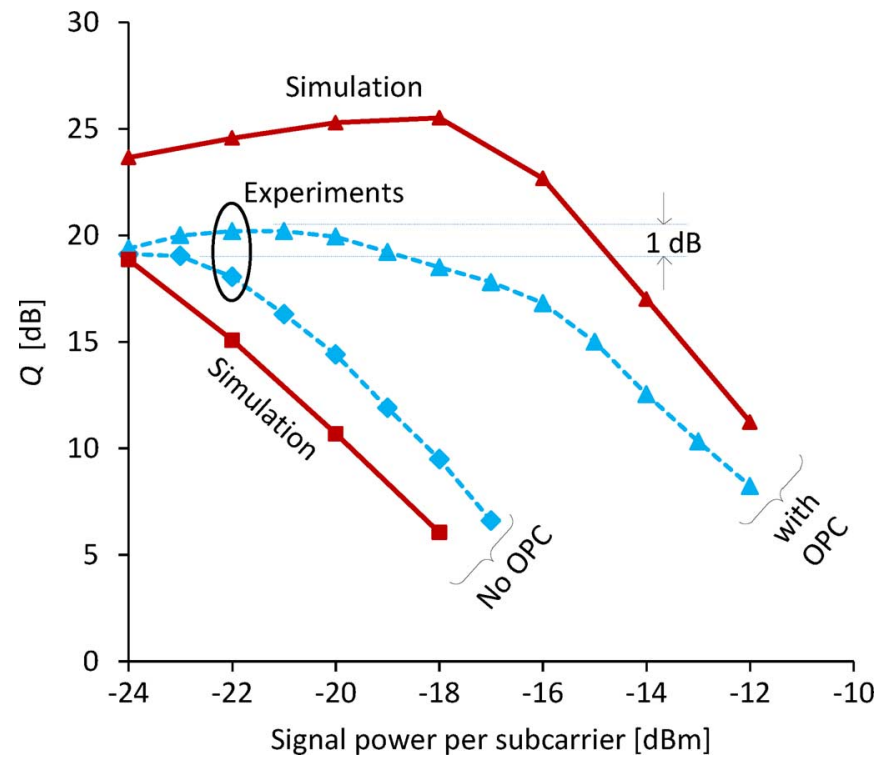

Fig. 11. Transmission performance over $800 \mathrm{~km}$ with and without OPC.

results without the OPC shift rightward, toward higher powers. On the other hand, in the systems with OPC, the experimental results show a lower NLT than the analysis. This is because ASE builds up along the link, reducing the signal power at the outputs of the later EDFAs. This results in an asymmetry in the nonlinear power distribution. This asymmetrical power in experiments degrades the nonlinear compensation effect of the OPC.

\section{CONCLUSION}

We have presented analytical expressions for the performance limiting factors in CO-OFDM systems with MSSI using OPC. We have identified that two different two-stage mixing products, XPM-OPC and FWM-OPC, are the limiting factors in the high power region for CO-OFDM systems using OPC, with XPM-OPC dominating. Our analytical results agree well with our SSFM simulations and experiments. Our results show that OPC could support signals with over $10 \mathrm{THz}$ bandwidths, though the dispersion of the nonlinear element would have to be carefully managed. We have provided experimental demonstration of MSSI in a CO-OFDM using a link of $800 \mathrm{~km}$. We have shown that the NLT is increased by over $6 \mathrm{~dB}$ by MSSI.

\section{ACKNOWLEDGMENT}

The authors would like to thank VPIphotonics.com for the use of VPItransmissionMaker.

\section{REFERENCES}

[1] S. L. Jansen, I. Morita, T. C. M. Schenk, and H. Tanaka, "121.9-Gb/s PDM-OFDM transmission with 2-b/s/Hz spectral efficiency over 1000 km of SSMF," J. Lightw. Technol., vol. 27, no. 3, pp. 177-188, Feb. 2009.

[2] H. Takahashi, A. Al Amin, S. L. Jansen, I. Morita, and H. Tanaka, "DWDM transmission with 7.0-bit/s/Hz spectral efficiency using $8 \times 65.1-\mathrm{Gbit} / \mathrm{s}$ coherent PDM-OFDM signals," in Proc. Opt. Fiber Commun. Conf., San Diego, CA, 2009, pp. 1-3. 
[3] B. J. C. Schmidt, Z. Zan, L. B. Du, and A. J. Lowery, "120 Gbit/s over 500-km using single-band polarization-multiplexed self-coherent optical OFDM," J. Lightw. Technol., vol. 28, no. 4, pp. 328-335, Feb. 2010.

[4] Q. Yang, Y. Tang, Y. Ma, and W. Shieh, "Experimental demonstration and numerical simulation of $107-\mathrm{Gb} / \mathrm{s}$ high spectral efficiency coherent optical OFDM,” J. Lightw. Technol., vol. 27, no. 3, pp. 168-176, Feb. 2009.

[5] Y. Ma, Q. Yang, Y. Tang, S. Chen, and W. Shieh, "1-Tb/s single-channel coherent optical OFDM transmission with orthogonal-band multiplexing and subwavelength bandwidth access," $J$. Lightw. Technol., vol. 28, no. 4, pp. 308-315, Feb. 2010.

[6] A. J. Lowery, L. B. Du, and J. Armstrong, "Performance of optical OFDM in ultralong-haul WDM lightwave systems," J. Lightw. Technol., vol. 25, no. 1, pp. 131-138, Jan. 2007.

[7] R.-J. Essiambre, G. J. Foschini, G. Kramer, and P. J. Winzer, "Capacity limits of information transport in fiber-optic networks," Phys. Rev. Lett., vol. 101, pp. 163901-1-163901-4, 2008.

[8] A. D. Ellis, J. Zhao, and D. Cotter, "Approaching the non-linear Shannon limit," J. Lightw. Technol., vol. 28, no. 4, pp. 423-433, Feb. 2010.

[9] D. Rafique and A. D. Ellis, "Impact of signal-ASE four-wave mixing on the effectiveness of digital back-propagation in $112 \mathrm{~Gb} / \mathrm{s}$ PM-QPSK systems," Opt. Exp., vol. 19, pp. 3449-3454, 2011.

[10] L. B. Du and A. J. Lowery, "Improved single channel backpropagation for intra-channel fiber nonlinearity compensation in long-haul optical communication systems," Opt. Exp., vol. 18, pp. 17075-17088, 2010.

[11] E. Ip and J. M. Kahn, "Compensation of dispersion and nonlinear impairments using digital backpropagation," J. Lightw. Technol., vol. 26, no. 20 , pp. $3416-3425$, Oct. 2008

[12] X. Li, X. Chen, G. Goldfarb, E. Mateo, I. Kim, F. Yaman, and G. $\mathrm{Li}$, "Electronic post-compensation of WDM transmission impairments using coherent detection and digital signal processing," Opt. Exp., vol. 16 , pp. 880-888, 2008.

[13] E. Ip, "Nonlinear compensation using backpropagation for polarization-multiplexed transmission," J. Lightw. Technol., vol. 28, no. 6, pp. 939-951, Mar. 2010

[14] A. J. Lowery, "Fiber nonlinearity mitigation in optical links that use OFDM for dispersion compensation," IEEE Photon. Technol. Lett., vol. 19 , no. 19 , pp. $1556-1558$, Oct. 2007.

[15] K. Kikuchi, M. Fukase, and S.-Y. Kim, "Electronic post-compensation for nonlinear phase noise in a $1000-\mathrm{km} \mathrm{20-Gbit/s} \mathrm{optical} \mathrm{QPSK}$ transmission system using the homodyne receiver with digital signal processing," in Proc. Opt. Fiber Commun. Conf./Nat. Fiber Opt. Eng. Conf., 2007, pp. 1-3.

[16] L. B. Du and A. J. Lowery, "Practical XPM compensation method for coherent optical OFDM systems," IEEE Photon. Technol. Lett., vol. 22, no. 5, pp. 320-322, Mar. 2010.

[17] L. B. Du and A. Lowery, "Pilot-based cross-phase modulation compensation for coherent optical orthogonal frequency division multiplexing long-haul optical communications systems," Opt. Lett., vol. 36, pp. 1647-1649, 2011.

[18] B. Inan, S. Randel, S. L. Jansen, A. Lobato, S. Adhikari, and N. Hanik, "Pilot-tone-based nonlinearity compensation for optical OFDM systems," in Proc. 36th Eur. Conf. Exhib. Opt. Commun., 2010, pp. 1-3.

[19] S. L. Jansen, D. van den Borne, B. Spinnler, S. Calabro, H. Suche, P. M. Krummrich, W. Sohler, G.-D. Khoe, and H. de Waardt, "Optical phase conjugation for ultra long-haul phase-shift-keyed transmission," J. Lightw. Technol., vol. 24, no. 1, pp. 54-64, Jan. 2006.

[20] P. Minzioni, F. Alberti, and A. Schiffini, "Techniques for nonlinearity cancellation into embedded links by optical phase conjugation," $J$. Lightw. Technol., vol. 23, no. 8, pp. 2364-2370, Aug. 2005.

[21] X. Liu, Y. Qiao, and Y. Ji, "Reduction of the fiber nonlinearity impairment using optical phase conjugation in $40 \mathrm{~Gb} / \mathrm{s} \mathrm{CO}-\mathrm{OFDM}$ systems," Opt. Commun., vol. 283, pp. 2749-2753, 2010.

[22] L. B. Du, M. M. Morshed, and A. J. Lowery, "604-Gb/s coherent optical OFDM over $800 \mathrm{~km}$ of S-SMF with mid-span spectral inversion," in OptoElectron. Commun. Conf., Seoul, Korea, 2012, pp. 41-42.

[23] L. B. Du, M. M. Morshed, and A. J. Lowery, "Fiber nonlinearity compensation for OFDM super-channels using optical phase conjugation," Opt. Exp., vol. 20, pp. 19921-19927, 2012.
[24] V. Pechenkin and I. J. Fair, "Analysis of four-wave mixing suppression in fiber-optic OFDM transmission systems with an optical phase conjugation module," IEEE/OSA J. Opt. Commun. Netw., vol. 2, no. 9 , pp. 701-710, Sep. 2010.

[25] A. J. Lowery, S. Wang, and M. Premaratne, "Calculation of power limit due to fiber nonlinearity in optical OFDM systems," Opt. Exp., vol. 15, pp. 13282-13287, 2007

[26] W. Shieh and C. Athaudage, "Coherent optical orthogonal frequency division multiplexing,” Electron. Lett., vol. 42, no. 10, pp. 587-588, May 2006.

[27] A. J. Lowery and J. Armstrong, "Orthogonal-frequency-division multiplexing for dispersion compensation of long-haul optical systems," Opt. Exp., vol. 14, pp. 2079-2084, 2006.

Monir Morshed was born in Tangail, Bangladesh, in 1974. He received the B.Eng. degree in electrical and electronics engineering in 1991 and the M.Eng. degree in 2001 both from the Tokyo Institute of Technology, Tokyo, Japan. He is currently working toward the Ph.D. degree in electrical and computer systems engineering in the Department of Electrical and Computer Systems Engineering, Monash University, Melbourne, Vic., Australia.

From 2001 to 2003, he was with Yokohama R\&D Center, Furukawa Electric Company Ltd., Japan, developing directly modulated uncooled distributed feedback lasers. From December 2003 to 2009, he was with MSC Software Corporation, Japan, as an Advisory Application Engineer.

Liang Bangyuan Du (S'08-M'12) was born in Shengyang, China, in 1985 $\mathrm{He}$ received the B.Sc. (with first class Hons.) and Ph.D. degrees in electrical and computer systems engineering from Monash University, Melbourne, Vic., Australia, in 2007 and 2012, respectively.

His research interests include fiber nonlinearity mitigation in long-haul systems, advanced modulation formats and multi-carrier transmission, including orthogonal frequency division multiplexing (OFDM). He is currently working at Monash University as a Research Fellow.

Dr. Du received Corning Outstanding Student Paper Award and the Optical Fiber Communication Conference in 2011 for his work on cross-phase modulation compensation and the Best Student Paper Award in the Transmission Systems subcommittee for work involving mid-span spectral inversion for optical OFDM

Arthur James Lowery (M'92-SM'96-F'09) was born in Yorkshire, U.K., in 1961. He received the B.Sc. degree (with first class Hons.) in applied physics from the University of Durham, Durham, U.K., in 1983, and the Ph.D. degree in electrical and electronic engineering from the University of Nottingham, Nottingham, U.K., in 1988.

From 1983 to 1984, he was with Marconi Radar Systems Ltd., U.K. In 1984, he joined the University of Nottingham and pioneered time-domain field modeling of semiconductor lasers as the transmission-line laser model. In 1996, he cofounded Virtual Photonics Pty. Ltd. (now VPIsystems Inc.) and led to the development of VPI's physical-level photonic design automation tools such as VPItransmissionMaker. In 2004, he became a Professor with the Department of Electrical and Computer Systems Engineering, Monash University, Melbourne, where he was the Head between 2007 and 2012. In 2008, he founded Ofidium to commercialize optical orthogonal frequency-division multiplexing (OFDM) for long-haul systems. He is currently the Director of the Monash Vision Group's Bionic Eye project and Science Leader in the Australian Research Council's Centre of Excellence for Ultrahigh Bandwidth Devices for Optical Systems. He has published more than 250 papers and four book chapters on the simulation of photonic devices and circuits and photonic applications such as mode-locking and optical transmission systems and nonlinearity compensation.

Dr. Lowery received the Peter Doherty Prize Award for Innovation, along with J. Armstrong and L. Ryan, for their technical work on optical OFDM in June 2006. In 2007, he received the Clunies Ross Award from the Australian Academy of Technological Sciences and Engineering (ATSE) for his work at VPIsystems. He is a Fellow of the ATSE. 